\title{
"UM MÉTODO DE MEDIDA DA EXPANSÃO TÉRMICA VOLUMÉTRICA DIFERENCIAL PARA AMOSTRAS CRISTALINAS DE DIMENSÕES REDUZIDAS*"
}

\author{
KLEMENSAS RIMGAUDAS JURAITIS ${ }^{a}$ \\ WALTER SANO \\ JOÃO BAPTISTA DOMICIANO ${ }^{a}$ \\ CLEMÊNCIO TEODORO DOTTO ${ }^{a}$ \\ EDUARDO DI MAUROa
}

\begin{abstract}
RESUMO
A técnica utilizada se baseia no trabalho de Thomas e col., na qual introduzimos algumas modificações devido às características especiais da amostra. Foi testado na determinação da transição de fase estrutural dó cristal de Cloreto de Níquel Dihidratado. As principais características deste cristal são tamanho reduzido, higroscópico e mecanicamente frágeis. Os valores experimentais obtidos concordam com os valores calculados teoricamente a partir dos dados estruturais de Raio-X, e o método é sensivel a $1 \%$ de variação de volume.
\end{abstract}

PALAVRAS-CHAVE: Expansão Volumétrica Diferencial; Cloreto de Níquel Dihidratado; Transição de Fase Estrutural.

\section{1 - INTRODUÇÃO}

Existem vários métodos absolutos GRAHAM et alii ${ }^{1}$ para medir a expansividade linear dos sólidos: Análise de Difraçâo de Raios-X, Franjas de Interferência da Luz, Variação da Capacitância Elétrica, etc. e também, pelo Método de Dilatação Térmica Diferencial. Este método utiliza um líquido onde a amostra é mergulhada, de modo que a dilatação da amostra se obtém através da medida das dilatações da amostra mais o líquido e subtraindo-se a dilatação do líquido. Embora o processo seja muito simples, exige uma escolha cuidadosa da substância utilizada como referência. A grande vantagem desse método reside no fato dele permitir a medida da expansão volumétrica total e não necessitar do conhecimento da forma geométrica da amostra e tanto pode ser utilizado um cristal único como vários deles, que o resultado final será o mesmo. O método experimental empregado no presente trabalho baseou-se no trabalho de THOMAS et alii ${ }^{2}$, com ligeiras modificações.

O cristal em estudo, Cloreto de Níquel Dihidratado $\left(\mathrm{NiCl}_{2} \cdot 2 \mathrm{H}_{2} \mathrm{O}\right)$, apresenta como características peculiares de que as amostras obtidas são de dimensões reduzidas (alguns microgramas), extremamente higroscópicas e muito frágeis a tração e compressão mecânicas. Dessa maneira o Método de Dilatação Térmica Diferencial é a escolha mais viável dentre os citados por ser o método mais simples que mantém inalterado o estado dos cristais durante as medidas. Com este processo obtivemos a curva de dilatação térmica volumétrica e caracterizarmos a região de transição de Fase Estrutural.
Para tanto, utilizamo-nos de vários cristais com um volume suficiente para obter uma boa precisão da variação volumétrica e testamos vários líquidos de referência até chegarmos a conclusão de que o álcool isopropílico é o que dá o melhor resultado.

Informações sobre a preparação dos cristais podem ser encontradas na tese de JURAITIS ${ }^{3}$, e sobre a sua estrutura cristalina no trabalho de MOROSIN ${ }^{4}$.

\section{2 - MÉTODO EXPERIMENTAL}

O método se baseia na obtenção da expansividade volumétrica em relação à temperatura. Seja

$$
\beta(T)=-\frac{1}{V} \frac{d V}{d T}
$$

visto que, para um sistema (liquido + cristal), o volume total é dado por $\mathrm{V}=\mathrm{Vl}+\mathrm{Vc}$, uma pequena variação na temperatura do sistema implica numa variaçāo do voume, ou seja:

$$
\frac{d V}{d T}=\frac{d V 1}{d T}+\frac{d V c}{d T}
$$

dividindo esta relação pelo volume total ellcontramos a expansividade do sistema: a.

b.

Instituto de Fisica - Universidade de São Paulo

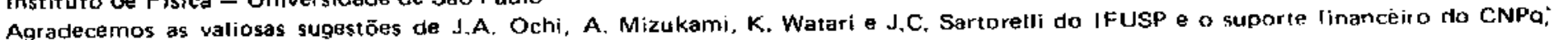
CAPES e a CPG/Universidade Estadual de Londrína. 


$$
\begin{aligned}
& 1 \mathrm{dV} \quad 1 \mathrm{dV} 1 \quad \mathrm{dVc} \\
& \bar{V}{ }_{\mathrm{dT}}=\frac{-}{\mathrm{V}}\left(\frac{---}{\mathrm{dT}}+\frac{---}{\mathrm{dT}}\right)
\end{aligned}
$$

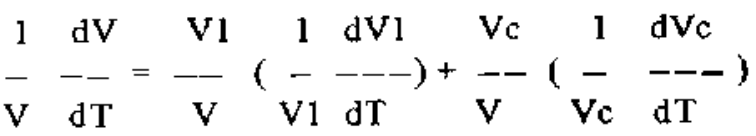

resultando, entāo, numa relaçāo entre as expansividades:

$$
\beta=\frac{\mathrm{V} 1}{\mathrm{v}} \beta 1+\frac{\mathrm{ve}}{\mathrm{v}} \beta c
$$

Assim sendo, o coeficiente de expansāo térmica do cristal será dado pela seguinte relação:

$$
\begin{aligned}
& \beta \mathrm{c}=\begin{array}{c}
\mathrm{V} \\
\mathrm{V1}
\end{array} \\
& \beta c=\frac{1}{V c}(V . \beta-V 1 . \beta 1) \\
& \beta c=\frac{1}{V_{c}} \quad\left(\begin{array}{cc}
d V & d V^{\prime} \\
-- & ---
\end{array}\right)
\end{aligned}
$$

e, dessa forma, obtemos a expressāo operacional:

$$
B c \quad=\frac{1}{V_{c}} \quad \frac{d}{d r}\left(V-v_{1}\right)
$$

Se medirmos a variação do volume do sistema (líquido + cristal) e a variação do volume do liquido em funçăo da temperatura, podemos encontras o coeficiente de expansāo térmica do crista!.

Na rnontagem experimental utilizamos um portaamostras de quartzo de volume aferido ligado a um tubo capilar também aferido. A união entre eles é feita por uma junta de latāo de modo a formar um corpo único, como se poule ver na figura 1 .

Coloca-se a amostra cristalina no tubo de quart $z 0$ e preenche-se carn um líquido de referencia adequado (neste caso o álcool isopropilico). A junta de lațo é dotada de um orifício através do qual é introduzido um termopar de Cobre Constantan sendo que este orificio ć lacrado posteriormente com araldite. O termopar tem um comprimento suficiente para que sua ponti repouse sobre o cristal, permitindo com isso determinar a temperatura em que se encontra o cristal e o líquido que a circunda. A junta de acoplamento é dotada de rosca e a sua vedação é feita através de um "O-ring”, permitindo, assim, um acesso à amostra no tubo de quartzo. Tanto o tubo de quartzo, quanto o capilar são colados nas respectivas peças de acoplamento com araldite.
Aquecendo-se ou resfriando-se o sistema lentamente, podemos obter a dilataçāo volumétrica relativa dos cristais, através da leitura da altura do menisco do líquido no tubo capilar, por meio de uma escala.

A altura do menisco é lida utilizando-se uma luneta telescópica de um catetómetro graduada em $0.1 \mathrm{~mm}$ e com precisāo de leitura da ordem de $0.05 \mathrm{~mm}$.

A variação da temperatura da amostra é acompanhada através de um registrador potenciométrico que, além de permitir a leitura da temperatura, fornece a indicaçăo do processo quasi-estático. $O$ Termopar tem por referéncia o gêlo fundente.

Para aquecer ou resfriar o sistema porta-amostras, utilizamos um "dewar" parcialmente preenchido com nitrogénio líquido. A temperatura adequada for obtida aproximando-se ou afastando-se o sistema da superficie do nitrogenio líquido. $O$ sistema entra em equilibrio em cerca de 15 minutos. Como o sistema é sensivel a vibraçoes, fixamos o tubo porta-amostras num suporte e movemos o "dewar" para cima ou para baixo através de um macaco de precisão. $O$ desenho esquemático da montagem exṕrimental pode ser visto na figura 2 .

O gradiente de temperatura jo longo do eixo vertical que passa pelo centro do "dewar", considerando-se desde a superfície do nitrogénio liquido até o gargalo do mesmo, mostrou-se linear em quase toda a sua extensao, como pode ser observado no gráfico da figura 3 . A taxa de evaporação do nitrogénio líquido tamberm foi determinada durante um tempo equivalente ao dà experiência, resultando ser suficientemente lenta, de forma a nao influir no equiIjbrio térmico do sistema porta-amostras, como se pode observar no grafico da figura 4 , $O$ resfriamento do sistema porta-amostras ocorre de uma maneira gradual, acompanhan. do o gradiente de temperaturas ao longo do eixo vertical do "dewar". A obtenção de temperaturas quasi-estáveis foi conseguido movendo-se o sistema porta-amostras ao longo do eixo vertical e aguardando o equilibrio térmico do sistema em cada uma das posiçōes próximas e sucessivas.

No processo de resfriamento não surgem problemas com correntes de conveção, que provocam o aparecimento de bolhas no liquido. No processo de aquecimento, entre. tanto, elas podem aparecer e para evita-las o aquecimento deve ser bastante lento.

A escolha do liquido de referència baseja-se nas seguintes condiçōes:

(i) O líquido deve ter bajxo ponto de solidificação, de manneira que possa cobrir a maior faixa possivel de temperaturas. No caso, deve abranger a regiao de transiçāo de fase, ou seja entre 200 e 220 graus absolutos K.

(ii) O liquido deve ter uma pressáo de vapor suficientemente baixa, para que a evaporação possa ser controlada e não interfira em demasia na obtenção das medidas.

(iii) O júquido deve ser inerte em relação à composição química do cristal; não deve alterar as suas propriedades físicas, e nāo deve dissolver a amostra,

O líquido de referēncia escolhido foi o álcool isopropúlico, por satisfazer as condiçōes acima descritas. $O$ único 
controle necessário deste líquido foi o da evaporação.

Considerando-se que o contacto do líquido com o ar se dá na extremidade superior do tubo capilar e que, portanto, a sua temperatura nesta região é a ambiente, podemos constatar que sua taxa de evaporação será constante ao longo do tempo, como se pode observar no gráfico da figura 5. A taxa de evaporação foi medida com base na variação da altura do menisco em relação ao tempo.

A escolha do tubo capilar baseou-se nas seguintes condições: (i) O diâmetro fosse o menor possível, sem que a tensão superficial do líquido interferisse demasiadamente na obtenção das medidas; (ii) Que as irregularidades do seu diâmetro estivessem dentro das flutuações do erro experimental. O tubo capilar escolhido foi de $50 \mathrm{~cm}$ de comprimento por $1.8 \mathrm{~mm}$ de diâmetro, $\mathrm{O}$ menisco apresentou uma superfície aproximadamente esféria, com um raio de $1 \mathrm{~mm}$. O tubo de quartzo que serviu de porta-amostras, tinha as seguintes medidas: $25 \mathrm{~cm}$ de comprimento por $4 \mathrm{~mm}$ de diâmetro interno.

\section{3 - OBTENÇÃO DOS DADOS}

Para se obter a curva de dilatação volumétrica, devemos inicialmente obter as seguintes medidas experimentais: (i) Curva de evaporação do líquido de referência.

(ii) Curva de dilatação do líquido de referência, através dos pares de dados de temperatura e altura da coluna do capilar.

(iii) Curva de dilatação do líquido de referência mais amostra, através dos pares de dados de temperatura e altura da coluna capilar.

A principal fonte de erros nessas medidas é a flutuação das condições de pressão e temperatura do ambiente, as quais influem diretamente na evaporação do líquido de referência.

A evaporação do líquido de referência na coluna capilar é levefnente alterada pela condução térmica do próprio líquido e pelas correntes de convecção que aparecem durante o aquecimento. A curva da temperatura em função do tempo, obtida através do registrador potenciométrico, apresenta a forma esperada, da lei de resfriamento ou aquecimento de Newton:

$$
\mathbf{d T} / \mathbf{d t}=-\mathrm{k}(\mathrm{T}-\mathrm{Tf})
$$

de modo que, através dessa curva, podemos controlar o processo termodinâmico de um modo quasi-estático, esperando quê o sistema entre em equilíbrio térmico. Integrando a expressão acima, obtemos:

$$
\mathrm{T}=\mathrm{To}+(\mathrm{Tf}-\mathrm{To})(1-\exp (\mathrm{k} \cdot \mathrm{t}))
$$

onde, To é a temperatura inicial, Tf é a temperatura final, t é o tempo de resfriamento ou aquecimento de To à $\mathrm{Tf}$ e $\mathrm{k}$ é uma constante proporcionalidade que depende da geometria e da natureza do sistema porta-amostras.

Os gráficos da figura 6 , mostram as curvas de resfriamento, onde estão anotados os valores de To, Tf e t, que, associados à medida $\mathrm{h}$ do capilar, fornecem os dados neces- sários para a obtenção da curva dilatométrica.

Tomando-se os dados de uma curva específica e fazendo-se o ajuste dos mesmos para a lei acima citada, verificamos uma boa concordância. A curva experimental e a curva ajustada podem ser vistas no gráfico da figura 7 .

Para se calcular a dilatação a partir dos pontos experimentais devemos primeiro descontar a evaporação. Feito isto, obtemos a curva de dilatação do líquido de referência em relação à temperatura. Pode-se observar no gráfico da figura 8 a curva de dilatação volumétrica do líquido de referência, com e sem o desconto da evaporação. Os pontos experimentais são ajustados numericamente através de uma regressão

No gráfico da figura 9, podemos observar a curva de dilatação em condições semelhantes para o sistema (líquido de referência mais cristal). Fazendo-se o desconto devido à dilatação, do líquido obtemos a variação volumétrica devida ao cristal:

$$
\mathrm{Vc}=\mathrm{V}-\mathrm{V} 1
$$

A curva obtida a partir desses dados está no gráfico da figura 10. Essa curva mostra a variação da altura do capilar, mas, como conhecemos a secção reta do mesmo, podemos calcular a variação volumétrica do cristal.

\section{4 - RESULTADOS EXPERIMENTAIS}

Como este método dilatométrico determina a expansão volumétrica e, portanto, não analisa a expansão em função dos parâmetros de rede, e como as nossas amostras consistem de cristais de tamanhos reduzidos, utilizamos vários cristais para aumentar o volume inicial da amostra a fim de obtermos um resultado significativo.

Foram utilizados $1,048 \mathrm{~g}$ de amostra cristalina em pó de Cloreto Níquel Dihidratado, correspondendo a um volume de $0.400 \mathrm{~cm}^{3}$ à temperatura ambiente. Este valor foi obtido a partir dos dados da densidade $(\mathrm{d}=2.622(2)$ $\mathrm{g} / \mathrm{cm}^{3}$ ) fornecida por MOROSIN ${ }^{4}$.

Baseando-se nos dados de Raios X (MOROSIN ${ }^{4}$, e BONGAARTS et alii $^{5}$ ) calculamos a variação de volume que deve ocorrer durante a transição de fase estrutural, a partir dos parâmetros de rede,

$$
a=6.9093 \mathrm{~A}, \mathrm{~b}=6.8858 \mathrm{~A}, \mathrm{c}=8.298 \mathrm{~A}, \beta=92.246 \underline{\mathrm{U}}
$$

para uma cela unitária de 4 unidades formula, acima da transição, e

$$
a^{3}=11.21(2) A, b^{\prime}=6.90(1) A, c^{3}=13.86(2) A, \beta=127.4(1) \underline{0}
$$

para cela unităria de 8 unidades fórmula, abaixo da transição, que foi a seguinte:

$$
\text { Variaçăo esperada }=0.00617(7) \mathrm{cm}^{3}
$$

A nossa experiência de dilataçāo volumétrica nos indicou que houve uma variaçāo de volume durante a transiçâo de fase, de: 
Variação experimental $=0.006(6) \mathrm{cm}^{3}$

o que mostra uma ótima concordância com o valor esperado. Vale notar que este valor representa uma variação de pouco mais que $1 \%$ do volume total da amostra o que atesta a sensibilidade deste método.

\section{5 - CONCLUSÃO}

Podemos ver também pelo gráfico da figura 10, que a transição ocorre por volta de $200 \mathrm{~K}$ no resfriamento e $220 \mathrm{~K}$ no aquecimento, concordando com os nossos resultados anteriores de Ressonância Paramagnética Eletrônica (JURAITIS et alií $^{6}$ ), bem como com os dados de Calor Específico (JURAITIS ${ }^{3}$ ), evidenciando a existência da histerese térmica. A alteração brusca de volume na temperatura de transição confirma que realmente a transição de fase é estrutural.

A concordância da variação volumétrica teórica obtida por nós a partir dos dados experimentais de raios-X de MOROSIN $^{4}$ e de BONGAARTS et alii ${ }^{5}$, com a variação volumétrica experimental está dentro do erro experimental o que justifica plenamente a viabilidade deste método para medidas de variação de volume de $1 \%$.

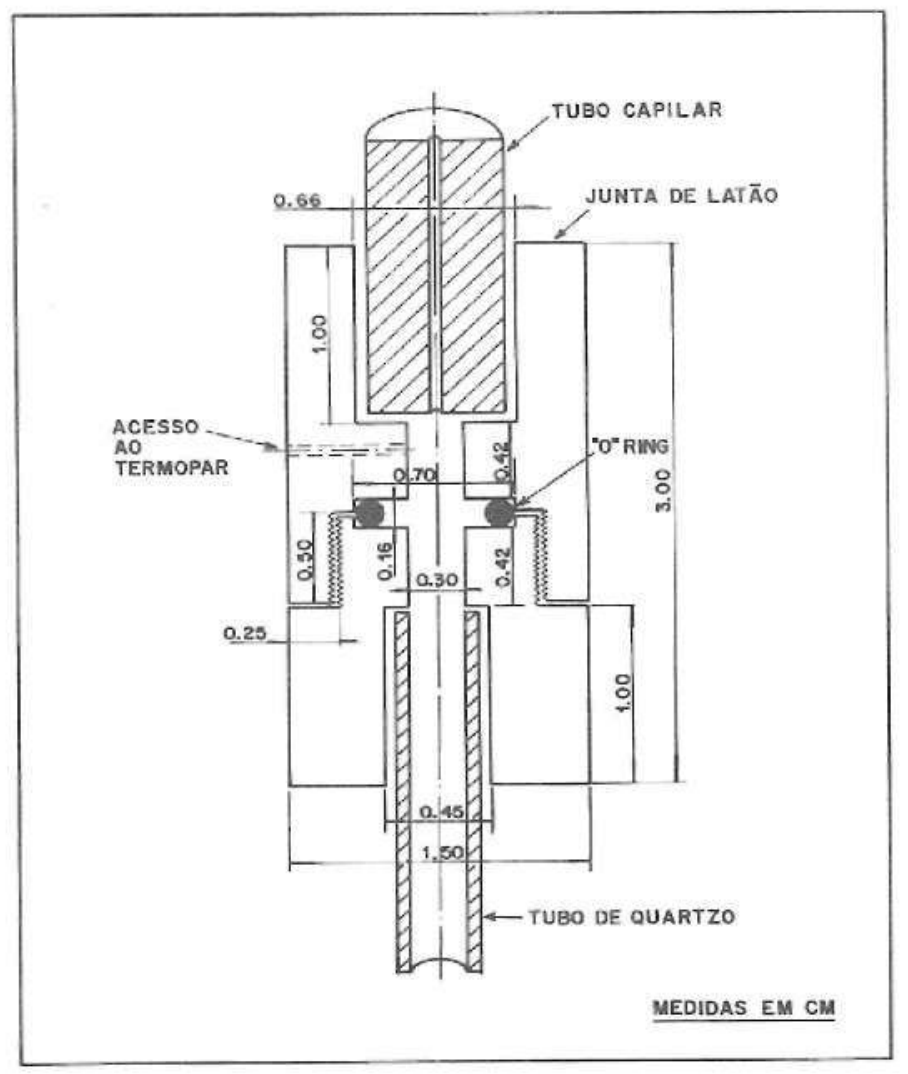

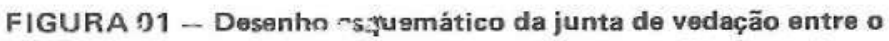
tulso porta-amostras e o tubo capilar do sistema dilatométrico.

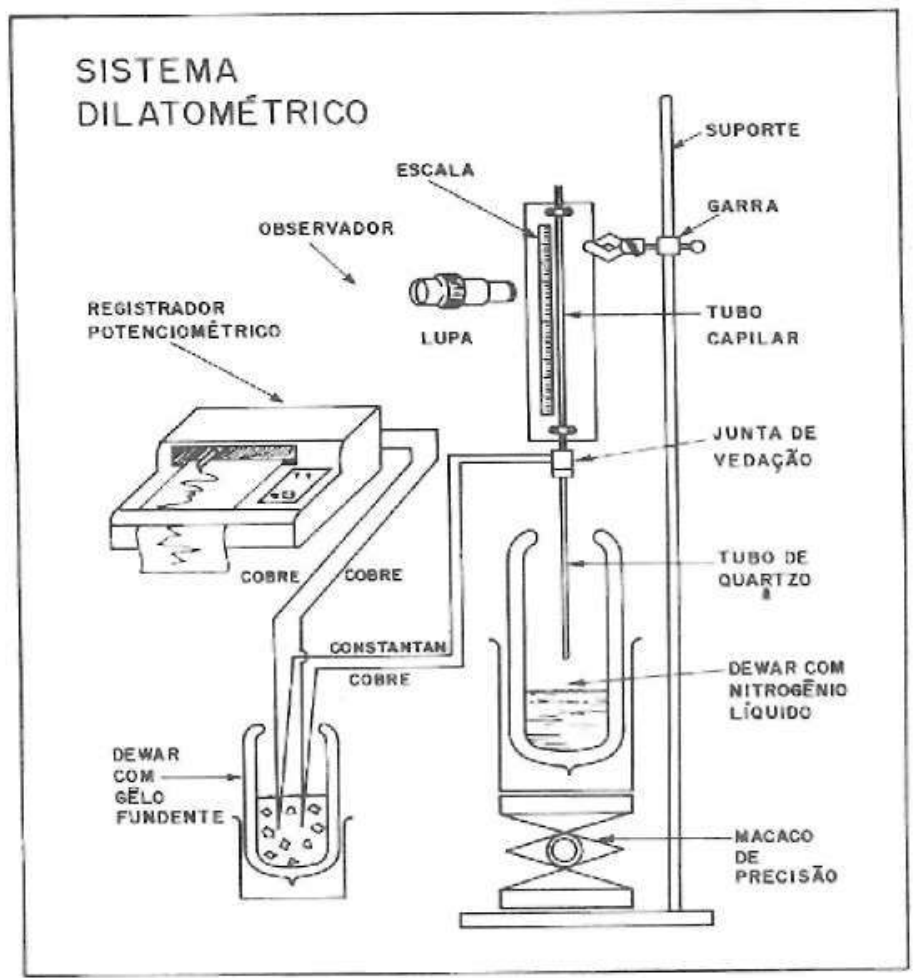

FIGURA 02 - Desenho esquemático da montagem experimental do sistema dilatom6trico

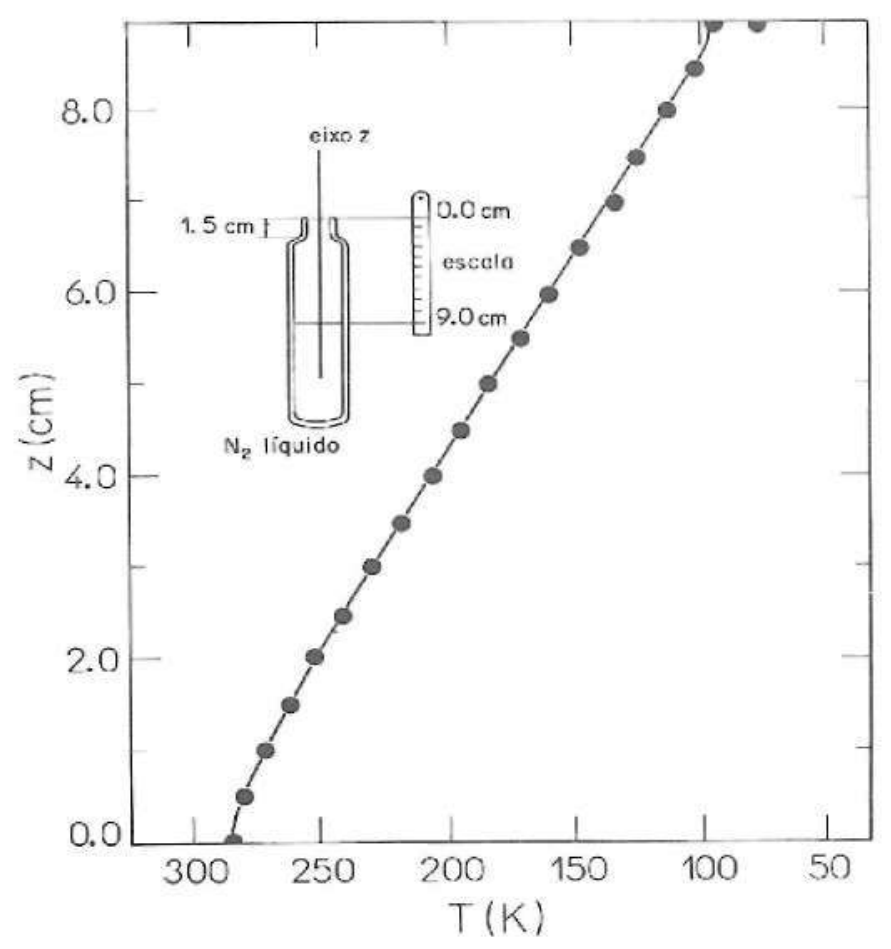

FIGURA 03 - Gráfico do gradiente de temperaturas ao longo do eixo de um dewar aberto, parcialmente preenchido com nitrogennio liquido 


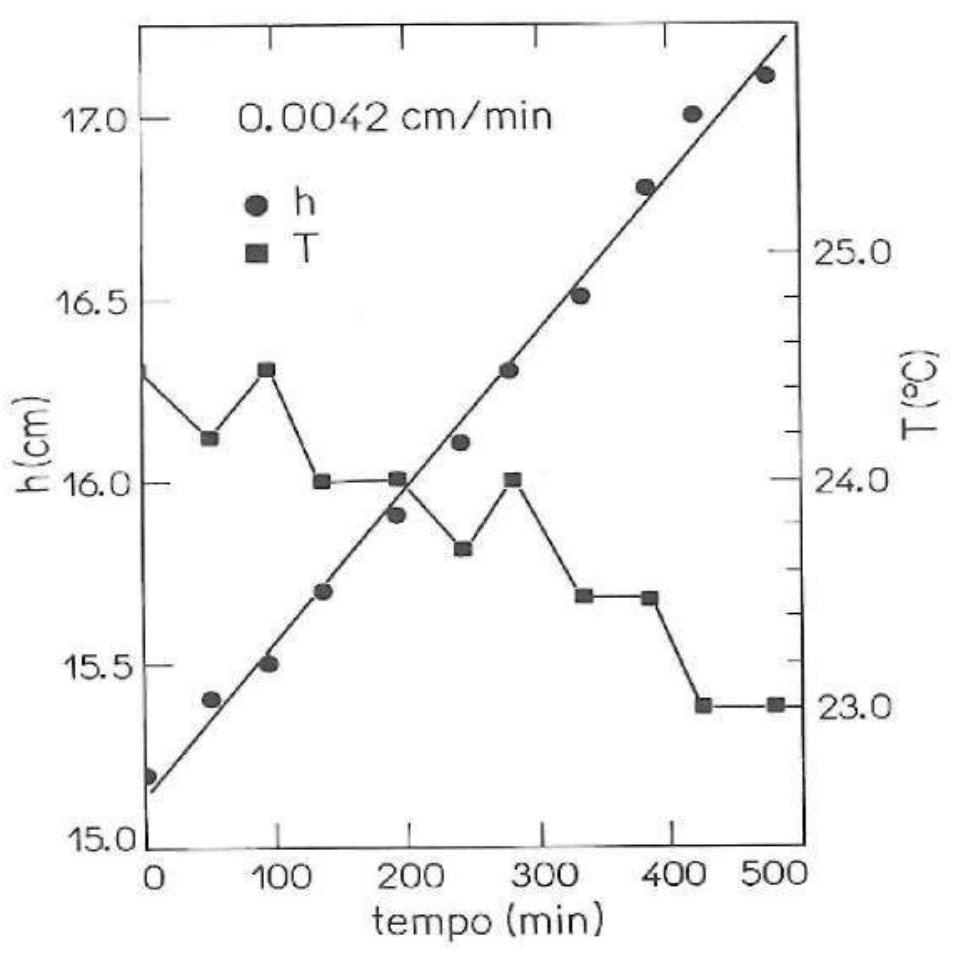

FIGURA 04 - Gráfico da taxa de evaporação do nitrogênio Ifquido no dewar aberto e a variação da temperatura ambiente

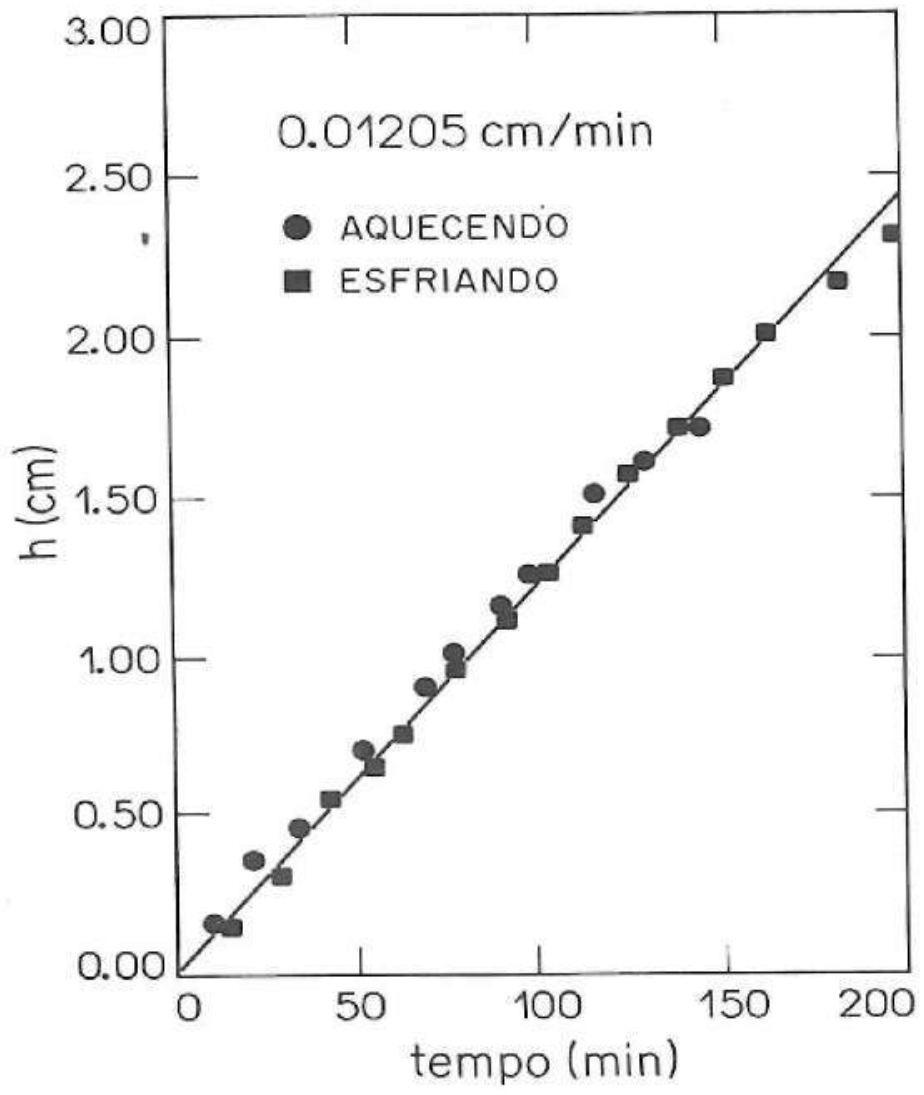

FIGURA 05 - Grafico da taxa de @vaporação do álcool isoprop flico no tubo capilar
CURVA DE RESFRIAMENTO

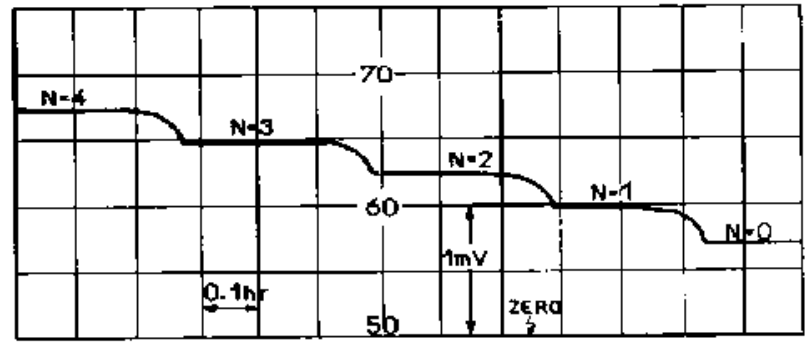

CURVA DE AQUECIMENTO

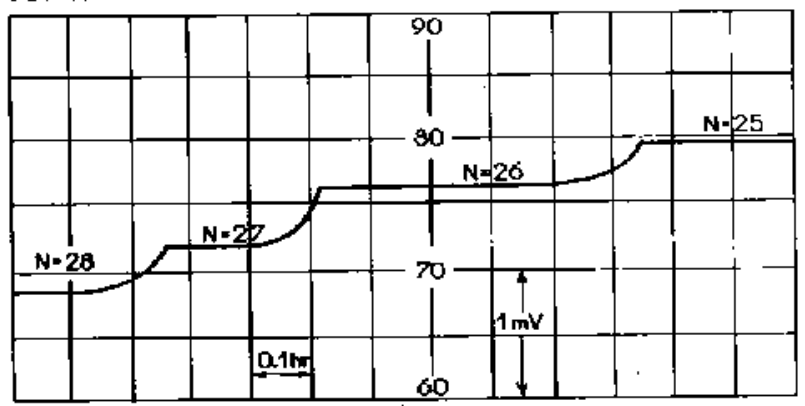

FIGURA 06:- Um exemplo das curvas de resfriamento e de aquecimento obtidas no registrador potenciometrico

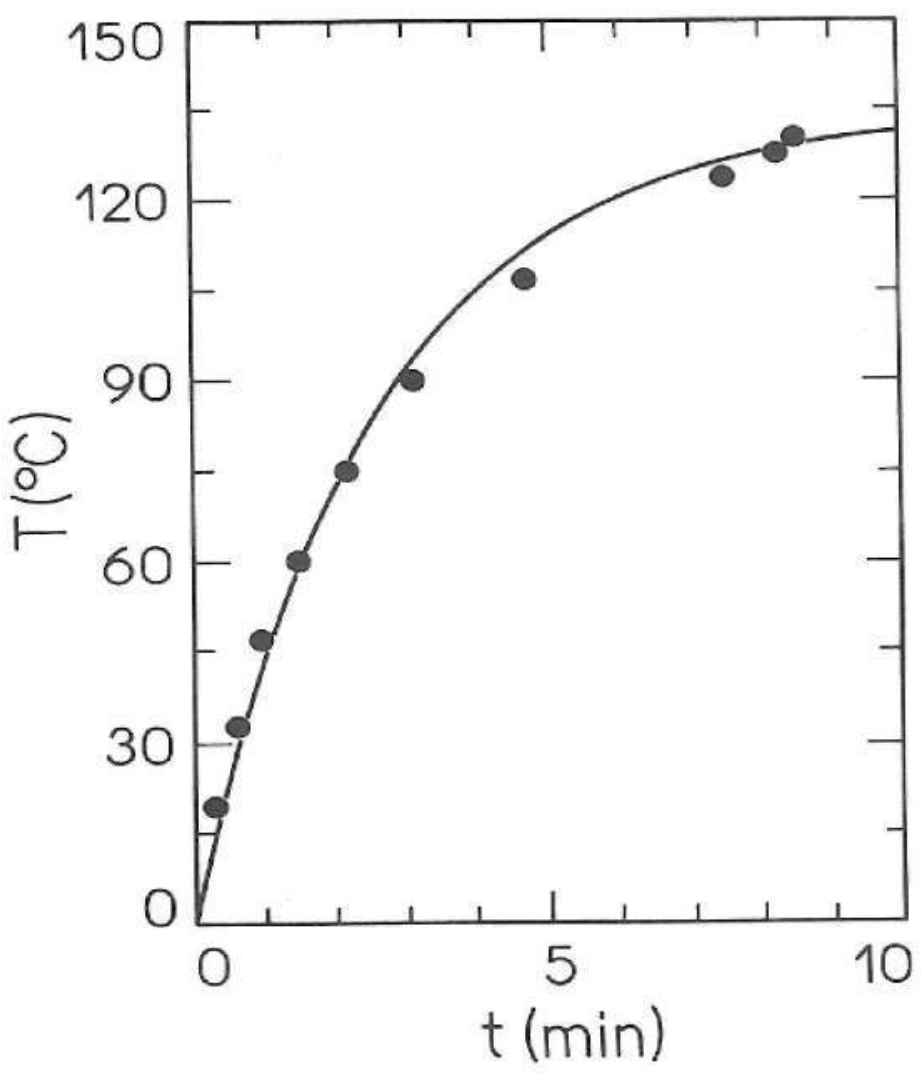

FIGURA 07 - Uma curva,de resfriamento ajustada segundo a lei de resfriamento de Newton 


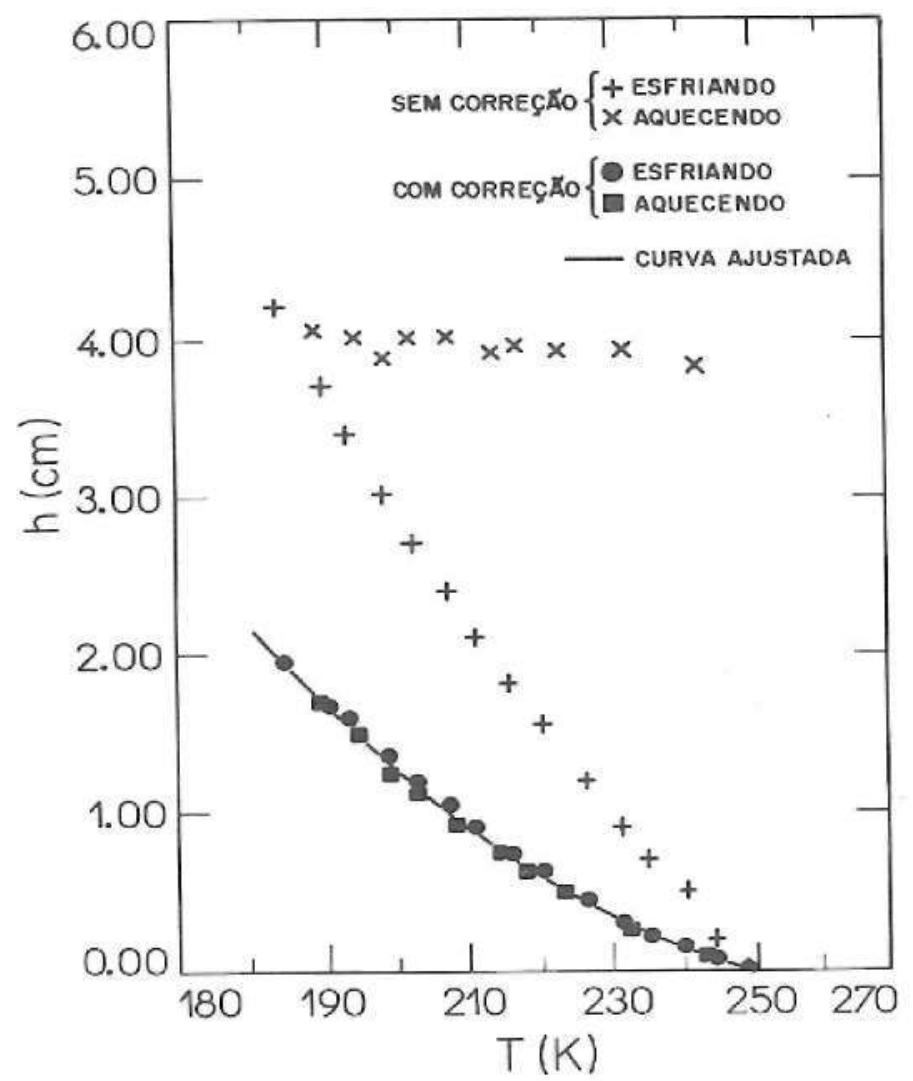

FIGURA 08 - Curva de dilatação volumétrica do álcool isoprop flico e o respectivo ajuste mediante o desconto da taxa de evaporação

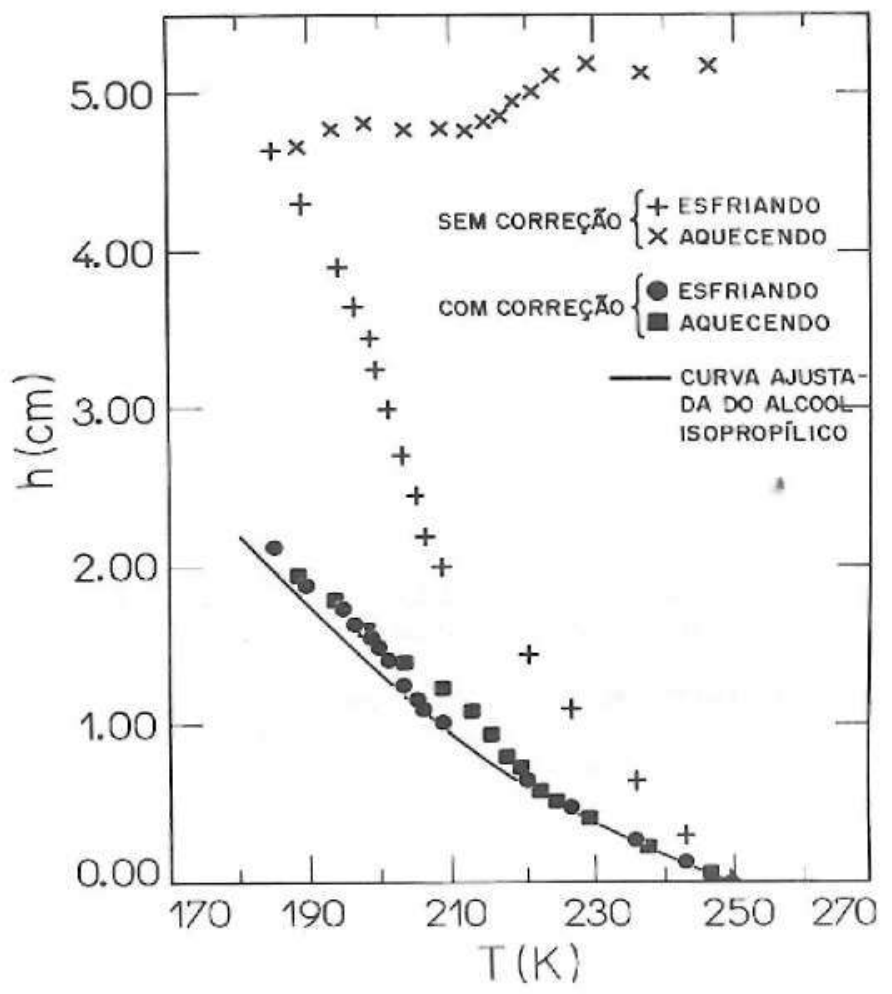

FIGURA 09 - Curva de dilatação volumétrica do sistema lálcool isoprop flico + cristel de clorêto de nfquel dihidratado 00 respectivo ajuste mediante o desconto da taxa de evaporação

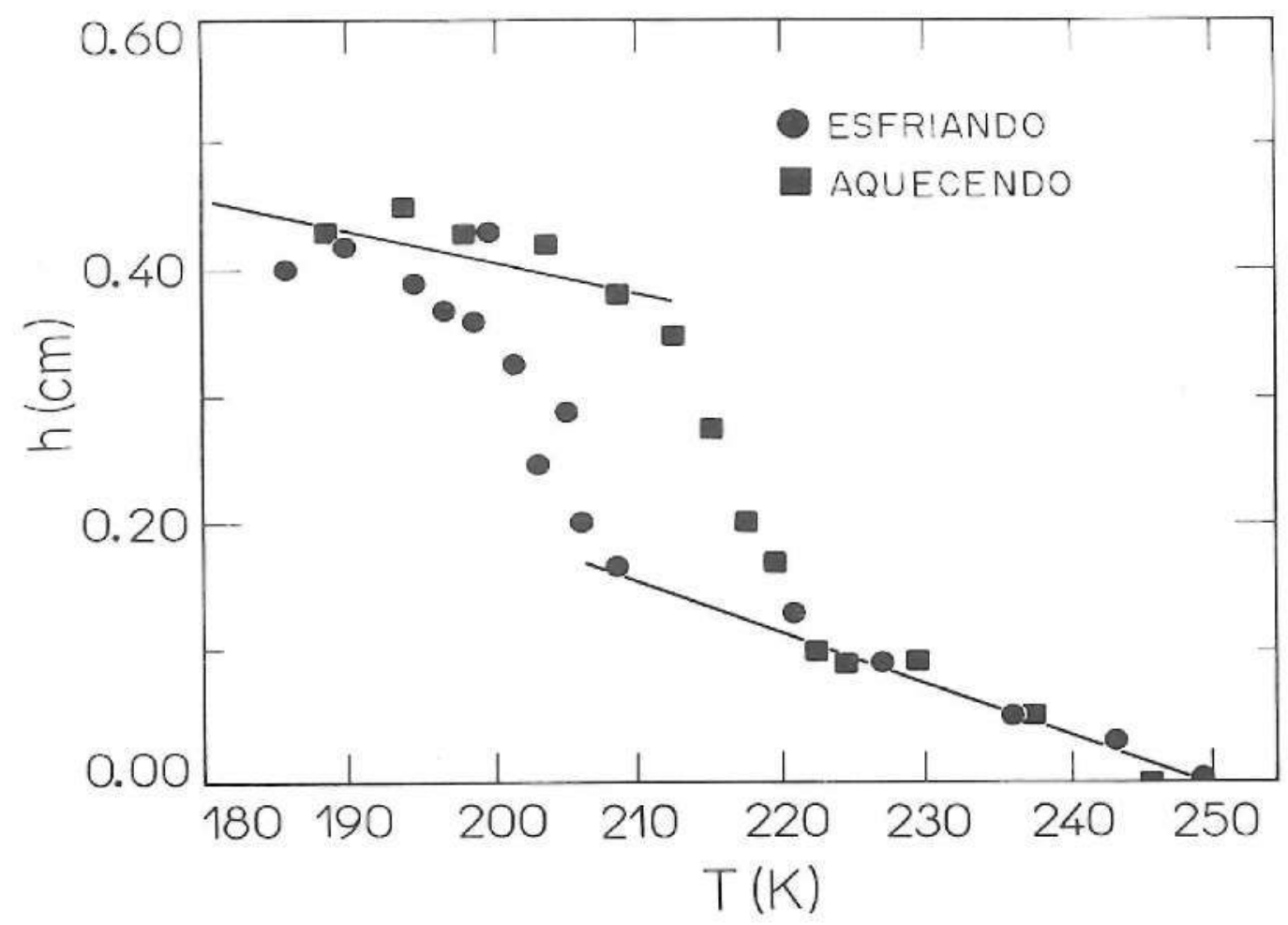

FIGURA 10 - Curva de dilatação volumétritea do cristal de clorêto de niquel dihidratario 


\begin{abstract}
In this paper a technique devised by Thomas and collaborators slightly modified to attend the special characteristics of the samples was apllied to determine the structural phase transition in crystals of $\mathrm{NiCl}_{2} \cdot 2 \mathrm{H}_{2} \mathrm{O}$. Small size, mechanical fragility and hygroscopic property were the main characteristics of these crystals. The experimental values obtained were in agreement with the theoretical calculations from structural data of $X$ Rays. The method is so precise that it indicates changes in $1 \%$ of volume variation.
\end{abstract}

KEY WORDS: Differential volumetric expansion; Dihydrated nickel chloride; Structural phase transition.

\title{
REFERENCIAS BIBLIOGRÁFICAS
}

1 - GRAHAM, M.G. \& HAGY, H.E. Thermal Expansion. Am. Inst. of Phys, 1972.

2 - THOMAS, D.G.; STAVELEY, L.A.K.; CULLIS, A.F. $J$. Chem. Soc.: 1727, 1952.

3 - JURAITIS, K.R. Estudo da transição de fase estrutural do Clorêto de niquel dihidratado com diferentes técnicas experimentais. São Paulo, IFUSP, 1985 Tese (Doutoramento).
4 - MOROSIN, B. An X-ray Diffraction Study on Nickel (II) Chloride Dihydrate. Acta Cryst., (23), 630, 1967.

5 - BONGAARTS, A.L.M.; Van LAAR, B.; BOTTERMAN, A.C.; De JONGE, W.J. Phase Transition in $\mathrm{NiCl}_{2} \cdot 2 \mathrm{H}_{2} \mathrm{O}$, Phys. Lett. (41A), 411, 1972.

6 - JURAITIS, K.R.; DOMICIANO, J.B.; SANO, W. Structural Phase Transition Study of $\mathrm{NiC}_{2} \cdot 2 \mathrm{H}_{2} \mathrm{O}$ by EPR. $J$. Phys. Chem. of Solids, 44(6), 531, 1983.

Recebido para publicação em 12/10/88 